\title{
A Note on Multiset Dimension and Local Multiset Dimension of Graphs
}

\author{
Ridho Alfarisi $^{1, *}$, Yuqing Lin ${ }^{2}$, Joe Ryan ${ }^{2}$, Dafik ${ }^{3}$, Ika Hesti Agustin ${ }^{4}$ \\ ${ }^{1}$ Department of Primary School, University of Jember, Indonesia \\ ${ }^{2}$ School of Electrical Engineering and Computing, University of Newcastle, Australia \\ ${ }^{3}$ Department of Mathematics Education, University of Jember, Indonesia \\ ${ }^{4}$ Department of Mathematics, University of Jember, Indonesia
}

\begin{abstract}
All graphs in this paper are nontrivial and connected simple graphs. For a set $W=\left\{s_{1}, s_{2}, \ldots, s_{k}\right\}$ of vertices of $G$, the multiset representation of a vertex $v$ of $G$ with respect to $W$ is $r(v \mid W)=\left\{d\left(v, s_{1}\right), d\left(v, s_{2}\right), \ldots, d\left(v, s_{k}\right)\right\}$ where $d\left(v, s_{i}\right)$ is the distance between of $v$ and $s_{i}$. If the representation $r(v \mid W) \neq r(u \mid W)$ for every pair of vertices $u, v$ of a graph $G$, the $W$ is called the resolving set of $G$, and the cardinality of a minimum resolving set is called the multiset dimension, denoted by $m d(G)$. A set $W$ is a local resolving set of $G$ if $r(v \mid W) \neq r(u \mid W)$ for every pair of adjacent vertices $u, v$ of a graph $G$. The cardinality of a minimum local resolving set $W$ is called local multiset dimension, denoted by $\mu_{l}(G)$. In our paper, we discuss the relationship between the multiset dimension and local multiset dimension of graphs and establish bounds of local multiset dimension for some families of graph.
\end{abstract}

Keywords Resolving set, Multiset Dimension, Local Resolving set, Local Multiset Dimension, Cartesian product, Hypercube Graph, Almost Hypercube Graph, Trees

\section{AMS 2010 subject classifications $05 \mathrm{C} 12$}

DOI: $10.19139 /$ soic-2310-5070-727

\section{Introduction}

All graphs discussed in this paper are finite, simple and connected graph. The cartesian product graph of $G_{1}$ and $G_{2}$, denoted by $G_{1} \times G_{2}$, is the graph with vertex set $V\left(G_{1}\right) \times V\left(G_{2}\right)$ where vertex $(x, u)$ is adjacent to vertex $(y, v)$ whenever $x y \in E\left(G_{1}\right)$ and $u-v$, or $x-y$ and $u v \in V\left(G_{2}\right)$. In the rest of the paper, we use the terminology defined in $[1,2,3]$. The application of metric dimension in networks is one of the describe navigation robots. The each place is called vertices and edges denote the connections between vertices. The minimum number of the robots required to locate each and the vertex of a some network is called as metric dimension, for more detail this application in [4].

The concept of metric dimension was independently introduced by Slater [5] and Harary and Melter [6]. In his paper, Slater called this concept the locating set. Let $u, v$ be two vertices in $G$, the distance $d(u, v)$ is the length of a shortest path between two vertices $u$ and $v$ in graph $G$. An ordered set $W=\left\{w_{1}, w_{2}, \ldots, w_{k}\right\}$ subset of vertex set $V(G)$. The representation $r(v \mid W)$ of $v$ with respect to $W$ is the ordered $k$-tuple $r(v \mid W)=\left(d\left(v, w_{1}\right), d\left(v, w_{2}\right), \ldots, d\left(v, w_{k}\right)\right)$. The set $W$ is called the resolving set of $G$ if every vertices of $G$ have distinct representation with respect to $W$. Let $u$ and $v$ be any two vertices in $G$ if $r(u \mid W)=r(v \mid W)$ implies that $u=v$. Hence if $W$ is a resolving set of cardinality $k$ for a graph $G$, then the representation set $r(v \mid W), v \in V(G)$ consists of $|V(G)|$ distinct $k$-vector. The minimum cardinality of resolving set of a graph $G$ is called metric dimension of $G$, denoted by $\operatorname{dim}(G)$.

\footnotetext{
${ }^{*}$ Correspondence to: Ridho Alfarisi (Email: alfarisi.fkip@ unej.ac.id). Department of Primary School, University of Jember. 37 Kalimantan
} Road, Jember, East Java, Indonesia (68121).

ISSN 2310-5070 (online) ISSN 2311-004X (print)

Copyright (C) 2020 International Academic Press 


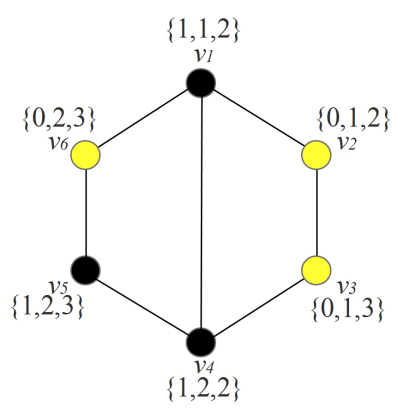

(a)

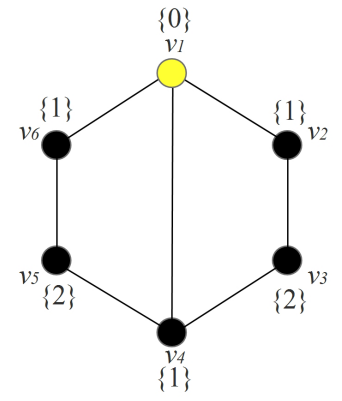

(b)

Figure 1. (a) A graph with multiset dimension 3; (b) A graph with local multiset dimension 1

Simanjuntak et al [7] introduced the definition of multiset dimension of $G$. Let $G$ be a connected graph with vertex set $V(G)$. Suppose $W=\left\{s_{1}, s_{2}, \ldots, s_{k}\right\}$ is a subset (note, not an ordered set as in metric dimension) of the vertex set $V(G)$, the representation multiset of a vertex $v$ of $G$ with respect to $W$ is the multiset $r(v \mid W)=\left\{d\left(v, s_{1}\right), d\left(v, s_{2}\right), \ldots, d\left(v, s_{k}\right)\right\}$ where $d\left(v, s_{i}\right)$ is the distances between of $v$ and the vertices in $W$ together with their multiplicities. A resolving set having minimum cardinality is called a multiset basis. If $G$ has a multiset basis, then its cardinality is called the multiset dimension of $G$, denoted by $\operatorname{md}(G)$. There are some related research about this topic in $[9,10,11]$.

Alfarisi, et. al [8] defined a new notion based on the multiset dimension of $G$, namely a local multiset dimension. The definition of local multiset dimension is below:

\section{Definition 1.1}

Let $G$ be a connected graph with vertex set $V(G)$. Suppose $W=\left\{s_{1}, s_{2}, \ldots, s_{k}\right\}$ is a subset of the vertex set $V(G)$, the representation multiset of a vertex $v$ of $G$ with respect to $W$ is $r(v \mid W)=\left\{d\left(v, s_{1}\right), d\left(v, s_{2}\right), \ldots, d\left(v, s_{k}\right)\right\}$, where $d\left(v, s_{i}\right)$ is a multiset of distances between of $v$ and the vertices in $W$ together with their multiplicities. The resolving set $W$ is a local resolving set of $G$ if $r(v \mid W) \neq r(u \mid W)$ for every pair of adjacent vertices $u, v$ of a graph $G$. The minimum local resolving set $W$ is called local multiset dimension, denoted by $\mu_{l}(G)$.

We illustrate this concept in Figure 1. In this case, the resolving set is $W=\left\{v_{2}, v_{3}, v_{6}\right\}$, shown in Figure 1 (a). The multiset dimension is $m d(G)=3$. The representations of $v \in V(G)$ with respect to $W$ are all distinct . For the local multiset dimension, we only need to make sure the adjacent vertices having distinct representations. Thus we could have the local resolving set $W=\left\{v_{1}\right\}$, shown in Figure 1 (b). Thus, the local multiset dimension is $\mu_{l}(G)=1$.

$$
\begin{array}{lll}
r\left(v_{1} \mid \Pi\right)=\{0\}, & r\left(v_{2} \mid \Pi\right)=\{1\}, & r\left(v_{3} \mid \Pi\right)=\{2\} \\
r\left(v_{4} \mid \Pi\right)=\{1\}, & r\left(v_{5} \mid \Pi\right)=\{2\}, & r\left(v_{6} \mid \Pi\right)=\{1\}
\end{array}
$$

\section{Multiset Dimension}

Different to the metric dimension, given a multiset basis, it is impossible to construct the original graph from the representation of the vertices. Fig 3 gives an example of two non-isomorphic graphs with the same multiset basis and representations for vertices.

\section{Lemma 2.1}

The multiset dimension is not monotonic to the number of vertices and the number of edges of a graph.

Let $G$ be a connected graphs. The number of vertices, edges and the multiset dimension do not show a monotonic relationship. Assume the graph $G$ with $n$ vertices has $m d(G)=k$, if we put $m$ vertices in graph $G$, then we get new graphs $G^{\prime}$ with $n+m$ vertices such that we have some condition as follows: 


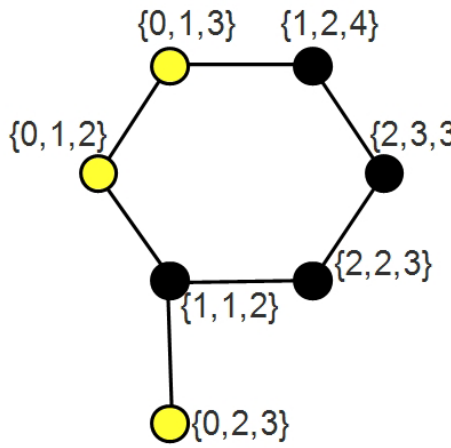

(a)

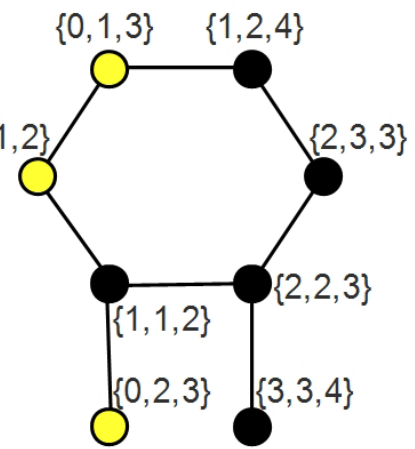

(b)

Figure 2. (a) $m d(G)=3$; (b) $m d(G \cup\{v\})=3$

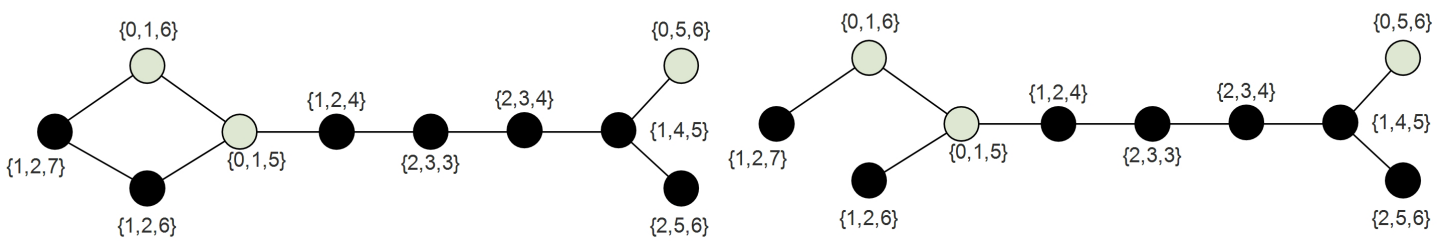

$\mathbf{G}_{1}$

$\mathbf{G}_{2}$

Figure 3. For $G_{1} \neq G_{2}$

i. if $G \cong G^{\prime}$, then $\operatorname{md}(G)=m d\left(G^{\prime}\right)=k$. (not monotonic)

ii. if $G \supsetneqq G^{\prime}$, then we have $m d(G) \geq m d\left(G^{\prime}\right)$ or $m d(G)<m d\left(G^{\prime}\right)$.

We use a counter example for showing the Lemma 2.1. Assume the number of vertices and edges of $G$ increase, the multiset dimension of $G$ increase or decrease (monotonically). We choose a unicyclic graph $G$ as example. From Figure 2 (b) we increase the vertices and edges in graph $G$ which have the multiset dimension of $G \cup\{v\}$ namely $\operatorname{md}(G \cup\{v\})=3$ where $v$ is a vertex not in the graph $G$. Furthermore, we can say that if we increase the number of vertices and edges in graphs $G$, then the number of resolving set does not increase or the multiset dimension is constant. it is a counter example of Lemma 2.1.

In Simanjuntak, et. al. [7], some bounds are given for the multiset dimension of graphs. For example,

Theorem 2.1

[7] Let $G$ be a graph other than a path. Then $\operatorname{md}(G) \geq 3$

If we look at the resolving set, since the vertex has distance 0 to itself, then it is easy to get a better bound than Theorem 2.2. For positive integers $n$ and $d$, we define $f(n, d)$ to be the least positive integer $k$ for which $\frac{(k+d 1) !}{k !(d 1) !}+k \geq n$.

Theorem 2.2

If $G$ is a graph of order $n \geq 3$ and diameter $d$, then $\operatorname{md}(G) \geq f(n, d)$

Proof. Let $W$ be a multiset basis of $G$ having $k$ vertices. If $x$ is a vertex in $W$, then $r(x \mid W)=$ $\left\{0,1^{m_{1}}, 2^{m_{2}}, \ldots, d^{m_{d}}\right\}$, where $m_{1}+m_{2}+\cdots+m_{d}=k-1$ and $0 m_{i} k$ for each $i=1,2, \ldots, k$. Then there are $C(k-1+d-2, d-2)$ different possibilities for representation of $x$. Since we have $k$ vertices in $W$, then $C(k-1+d-2, d-2)$ must be at least $k$.

Furthermore, look at the degree of the graph, we could have the following bounds. 
Theorem 2.3

Let $G$ be a connected graphs and let $d$ be the maximum degree of $G$ and $m d(G)=k$, we have for $k \geq 3$ and $d<3 k$.

Proof. As $\operatorname{md}(G)=k$, so let $W$ be a multiset basis of $G$ having $k$ vertices. If $x$ is a vertex not in $W$, then $r(x \mid W)=\left\{0,1^{m_{1}}, 2^{m_{2}}, \ldots, d^{m_{d}}\right\}$, where $m_{1}+m_{2}+\cdots+m_{d}=k$ and $0 \leq m_{i} \leq k$ for each $i=1,2, \ldots, k$. As $W$ is a minimum resolving set, removing a vertex from $W$, there will be two vertices in the graph $G$ which have the same representation. Let's assume that there are two vertices $v_{x}$ and $v_{y}$ which are in $G$. Formally, we have $r\left(v_{x} \mid W\right) \cap r\left(v_{y} \mid W\right)=\left\{1^{m_{1}}, 2^{m_{2}}, \ldots, d^{m_{d}}\right\}$, where $m_{1}+m_{2}+\cdots+m_{d}=k-1$. Considering the neighbour of these two vertices, if $v_{x}$ has distance $t$ to a vertex $w \in W$, then the neighbour of $v_{x}$ would have distance $t-1$ or $t$ or $t+1$ to $w$. So, if $v_{x}$ has distance 1 to $m_{1}$ vertices in $W$, then among $v_{x}$ 's neighbour, there are at most $m_{1}$ vertices having distance 0 to vertices in $W$, i.e. in $W$. if $v_{x}$ has distance 2 to $m_{2}$ vertices in $W$, then among $v_{x}$ 's neighbour, there are at most $m_{2}$ vertices having distance 1 to vertices in $W$, furthermore, $v_{x}$ 's neighbour having distance less than 3 to at least $m_{2}$ vertices in $W$. More general, if $v_{x}$ having distance $x$ to $m_{x}$ vertices in $W$, then among the neighbours of $v_{x}$, there are less than $m_{x}$ vertices of distance $x-1$ to $W$ and there are no more than $m_{x}$ vertices having distance $x+1$ to vertices in $W$.

Thus, the number of different representations for the neighbour of $v_{x}$ is $m_{1}\left(m_{1}+m_{2}\right)\left(m_{2}+m+3\right) . .\left(m_{d-1}+\right.$ $m d) d$ and the representations are shared by neighbours of $v_{x}$ and $v_{y}$. This will give us a bound for the degree, diameter and multiset dimension.

We shall define a new graph which is based on the well-known Hypercubes. The Hypercube is defined the graph formed from the vertices and edges of an n-dimensional hypercube, we shall remove some edges from the hypercube, denoted by $A H Q_{n}$, is called almost hypercube graphs. Almost hypercube graph satisfies $A H Q_{n}=$ $\left(H Q_{n-1} \times P_{2}\right)-\{e\}$ for $n \geq 3$, where $e$ is correspondence edge of subgraph $\left(H Q_{n-1}\right)_{1}$ and $\left(H Q_{n-1}\right)_{2}$. We know that $H Q_{n-1} \times P_{2}$ has two isomorphic graphs $\left(H Q_{n-1}\right)_{1}$ and $\left(H Q_{n-1}\right)_{2}$ with $\{e\}$ is the correspondence edge set.

Theorem 2.4

$m d\left(A H Q_{n}\right) \geq 2^{n-1}-1$, for $n \geq 3$

Proof: The cardinality of vertex set of almost hypercube graphs, denoted by $A H Q_{n}$, is $2^{n}$ for $n \geq N \cup\{0\}$. We can prove $m d\left(A H Q_{n}\right) \geq 2^{n-1}-1$.

Case 1: For $n=3$, we know that $m d\left(A H Q_{3}\right) \geq 3, m d\left(A H Q_{3}\right)>3$. If the resolving set of $A H Q_{3}$ is 2 , then we have some condition for position resolving set in $A H Q_{3}=\left(H Q_{1} \times P_{2}\right)-\{e\}$ in the following.

i. If three vertices in $H Q_{2}^{\prime}$, then there is at least two vertices which have same representation.

ii. If two vertices in $H Q_{2}^{\prime}$ and one vertex in $H Q_{2}$ ", then always two vertices with respect to $v \in W\left(H Q_{2} "\right)$ which have same representation.

Based on cases above, we know that $m d\left(A H Q_{3}\right) \geq 3$

Case 2: For $n=k$, we know that $m d\left(A H Q_{k}\right) \geq 2^{k-1}-1, m d\left(A H Q_{k}\right)>2^{k-1}-1$. If the resolving set of $A H Q_{k}$ is $2^{k-1}-2$, then we have some condition for position resolving set in $A H Q_{k}=\left(H Q_{k-1} \times P_{2}\right)-\{e\}$. We have some condition for proof this cases which divided into some cases as follows.

i. Shortest path between two vertices in the same components is within the component

ii. If $2^{k-1}-2$ vertices in $H Q_{n-1}^{\prime}$, then there is at least two vertices which have same representation. Let $v_{1}, v_{2} \in V\left(A H Q_{n}\right)$ be a correspondence vertices of $v_{1} \in V\left(H Q_{n-1}^{\prime}\right)$ and $v_{2} \in V\left(H Q_{n-1}\right.$ ") such that $d\left(v_{1}, r\right) \neq d\left(v_{2}, r\right)=d\left(v_{1}, r\right)+1$ for $r \in W$. We assume that the representation of $v_{1}$ and $v_{2}$ respect to resolving set $W$ namely $r\left(v_{1} \mid W\right)=\left\{d_{1}, d_{2}, d_{3}, \ldots, d_{x-3}, d\left(v_{1}, s\right)+1, d\left(v_{1}, s\right)\right\}$ and $r\left(v_{2} \mid W\right)=$ $\left\{d_{1}, d_{2}, d_{3}, \ldots, d_{x-3}, d\left(v_{1}, s\right), d\left(v_{2}, s\right)\right\}$ such that $r\left(v_{1} \mid W\right)=r\left(v_{2} \mid W\right)$, it is contradiction.

iii. If $2^{k-2}$ vertices in $H Q_{n-1}^{\prime}$ and $2^{k-2}-2$ vertex in $H Q_{n-1}$ ", then always two vertices with respect to $v \in W\left(H Q_{n-1} "\right)$ which have same representation. Let $v_{1}, v_{2} \in V\left(H Q_{n-1}^{\prime}\right)$ be a vertex in first position $H Q_{n-1}^{\prime}$ which have same representation respect to resolving set in $H Q_{n-1}^{\prime}$ namely $r\left(v_{1} \mid W^{\prime}\right)=r\left(v_{2} \mid W^{\prime}\right)=$ $\left\{d_{1}, d_{2}, d_{3}, \ldots, d_{\alpha-l}\right\}$. The distance of $v_{1}$ and $v_{2}$ respect to resolving set in second position $H Q_{n-1}$ " is symmetric distance such that $d\left(v_{1}, r\right)=d\left(v_{2}, r\right)$. Thus, we have the representation $v_{1}, v_{2} \in A H Q_{n}$ namely $r\left(v_{1} \mid W\right)=r\left(v_{2} \mid W\right)=\left\{d_{1}, d_{2}, d_{3}, \ldots, d_{\alpha-l}, d_{\alpha-l+1}, \ldots, d_{\alpha}\right\}$, it is contradiction. 


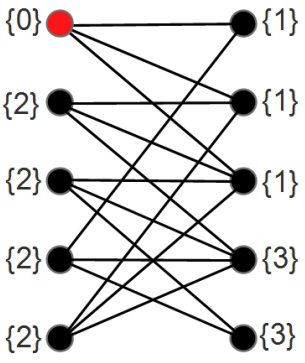

(a)

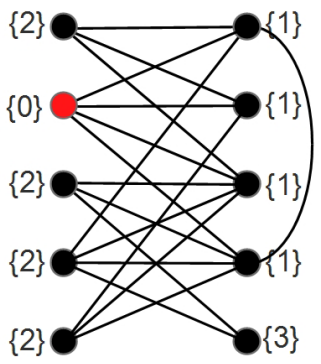

(b)

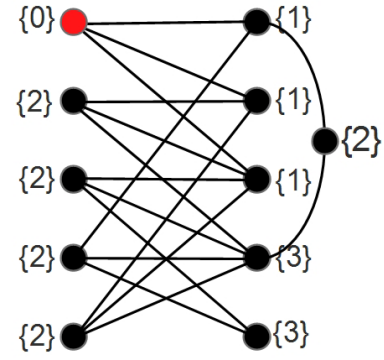

(c)

Figure 4. (a) $\mu_{l}\left(B_{5,5}\right)=1$; (b) $\mu_{l}\left(B_{5,5} \cup\{e\}\right)=1$; (c) $\mu_{l}\left(B_{5,5} \cup v\right)=1$

Based on both cases, we obtain the bounds of multiset dimension of almost hypercube graph namely $m d\left(A H Q_{n}\right) \geq 2^{n-1}-1$.

\section{Local Multiset Dimension}

In this section, we give some results about local multiset dimension of graphs. Firstly, we show that the local multiset dimension for AHQ is 1 , which is different to the results we got for multiset dimension.

Corollary 3.1

The difference between Multiset dimension and local multiset dimension can be arbitrarily large.

Observation 3.1

If $G$ is complete graph, then the graph $G$ does not have a local resolving set.

\section{Lemma 3.1}

The local multiset dimension is not monotonic to the number of vertices and the number of edges of a graph.

Let $G$ be a connected graphs. The number of vertices, edges and the local multiset dimension do not show a monotonic relationship. Assume the graph $G$ with $n$ vertices has $\mu_{l}(G)=k$, if we put $m$ vertices in graph $G$, then we get new graphs $G^{\prime}$ with $n+m$ vertices such that we have some condition as follows:

i. if $G \cong G^{\prime}$, then $\mu_{l}(G)=\mu_{l}\left(G^{\prime}\right)=k$. (not monotonic)

ii. if $G \supsetneqq G^{\prime}$, then we have $\mu_{l}(G) \geq \mu_{l}\left(G^{\prime}\right)$ or $\mu_{l}(G)<\mu_{l}\left(G^{\prime}\right)$.

We use a counter example for showing the Lemma 3.1. Assume the number of vertices and edges of $G$ increase, the local multiset dimension of $G$ increase or decrease (monotonically). We choose a bipartite graph $B_{5,5}$ as example. From Figure 4 (a) the local multiset dimension of $B_{5,5}$ is $\mu_{l}\left(B_{5,5}\right)=1$, we add some edges in $B_{5,5}$ in Figure 4 (b) such that we have the local multiset dimension of $B_{5,5} \cup\{e\}$ namely $\mu_{l}\left(B_{5,5} \cup\{e\}\right)=1$. Figure 4 (c) we increase the vertices and edges in graph $B_{5,5}$ which have the local multiset dimension of $B_{5,5} \cup\{v\}$ namely $\mu_{l}\left(B_{5,5} \cup\{v\}\right)=1$ where $v$ is a vertex not in the graph $B_{5,5}$. Furthermore, we can say that if we increase the number of vertices and edges in graphs $B_{5,5}$, then the number of resolving set does not increase or the local multiset dimension is constant. it is a counter example of Lemma 3.1.

The following, we show the new bound of local multiset dimension of cartesian product of graphs. Let $G_{1}$ and $G_{2}$ be two connected graphs.

\section{Lemma 3.2}

Given that two connected graphs $G_{1}$ and $G_{2}, \mu_{l}\left(G_{1} \times G_{2}\right) \geq \min \left\{\mu_{l}\left(G_{1}\right), \mu_{l}\left(G_{2}\right)\right\}$

Proof. A graph $G_{1}$ has $n_{1}$ vertices and $G_{2}$ has $n_{2}$ vertices. The cartesian product graph of $G_{1}$ and $G_{2}$, denoted by $G_{1} \times G_{2}$, is the graph with vertex set $V\left(G_{1}\right) \times V\left(G_{2}\right)$ where vertex $(x, u)$ is adjacent to vertex $(y, v)$ whenever 
$x y \in E\left(G_{1}\right)$ and $u-v$, or $x-y$ and $u v \in V\left(G_{2}\right)$. For a fixed $x$ of $G_{1}$, the vertices $\left\{(x, u) \mid u \in V\left(G_{2}\right)\right\}$ induces a subgraph of $G_{1} \times G_{2}$ isomorphic to $G_{2}$ and we call it as $G_{2}$-layer. Such that, we have $G_{1}$-layers or $G_{2}$-layers. Assume that we have local multiset dimension of $G_{1}$ and $G_{2}$, respectively are $\mu_{l}\left(G_{1}\right)=k_{1}$ and $\mu_{l}\left(G_{2}\right)=k_{2}$.

Case 1: If we choose $k_{1} \leq k_{2}$, such that we have $\left|W\left(G_{1}\right)\right|=k_{1}$. The position of resolving set in first layer of subgraph $G_{2}$, denoted by $\left(G_{2}\right)_{1}$, so we have distinct vertex representation in $\left(G_{2}\right)_{1}$. For the vertex in $\left(G_{2}\right)_{2}$ adjacent to the vertex in $\left(G_{2}\right)_{1}$, we have ford $d_{\left(G_{1} \times G_{2}\right)}\left(\left(x_{j}, u\right),\left(x_{1}, u^{*}\right)\right)=$ $d_{\left(G_{1} \times G_{2}\right)}\left(\left(x_{j}, u\right),\left(x_{1}, u\right)\right)+d_{\left(\left(G_{2}\right)_{1}\right)}\left(\left(x_{1}, u\right),\left(x_{1}, u^{*}\right)\right) \quad$ or $\quad d_{G_{1} \times G_{2}}\left(\left(x_{j}, u\right),\left(x_{1}, u *\right)\right)>d_{\left(G_{2}\right)_{1}}\left(\left(x_{1}, u\right),\left(x_{1}, u^{*}\right)\right)$. Such that for two adjacent vertices of vertex in $\left(G_{2}\right)_{1}$ and $\left(G_{2}\right)_{j}$ have distinct representation. For two adjacent vertex $\left(x_{1}, u_{i}\right),\left(x_{1}, u_{l}\right) \in V\left(\left(G_{2}\right)_{1}\right) \quad$ where $\quad d_{\left(G_{2}\right)_{1}}\left(\left(x_{1}, u_{i}\right),\left(x_{1}, u^{*}\right)\right) \neq d_{\left(G_{2}\right)_{1}}\left(\left(x_{1}, u_{i}\right),\left(x_{1}, u^{*}\right)\right)$. If we have two adjacent vertices in $\left(G_{2}\right)_{j}$-layers for $\left(x_{j}, u_{i}\right),\left(x_{j}, u_{l}\right) \in V\left(\left(G_{2}\right)_{j}\right)$ such that we have $d_{G_{1} \times G_{2}}\left(\left(x_{j}, u_{i}\right),\left(x_{1}, u^{*}\right)\right)=d_{G_{1} \times G_{2}}\left(\left(x_{j}, u_{i}\right),\left(x_{1}, u_{i}\right)\right)+d_{\left(G_{2}\right)_{1}}\left(\left(x_{1}, u_{i}\right),\left(x_{1}, u^{*}\right)\right) \quad$ and $d_{G_{1} \times G_{2}}\left(\left(x_{j}, u_{l}\right),\left(x_{1}, u^{*}\right)\right)=d_{G_{1} \times G_{2}}\left(\left(x_{j}, u_{l}\right),\left(x_{1}, u_{l}\right)\right)+d_{\left(G_{2}\right)_{1}}\left(\left(x_{1}, u_{l}\right),\left(x_{1}, u^{*}\right)\right) \quad$ and $\quad$ we $\quad$ know that $d_{G_{1} \times G_{2}}\left(\left(x_{j}, u_{i}\right),\left(x_{1}, u^{*}\right)\right) \neq d_{G_{1} \times G_{2}}\left(\left(x_{j}, u_{l}\right),\left(x_{1}, u^{*}\right)\right)$ for $\left(x_{1}, u^{*}\right) \in W \subset V\left(\left(G_{2}\right)_{1}\right)$.

Case 2: If we choose $k_{2}<k_{1}$, such that we have $\left|W\left(G_{2}\right)\right|=k_{2}$. The position of resolving set in first layer of subgraph $G_{1}$, denoted by $\left(G_{1}\right)_{1}$, so we have distinct vertex representation in $\left(G_{1}\right)_{1}$. From Case 1, we have same characterization of the vertex representation.

Based on both cases, we can claim that $\left|W\left(G_{1} \times G_{2}\right)\right|=k_{1}$ for $k_{1} \leq k_{2}$ and $\left|W\left(G_{1} \times G_{2}\right)\right|=k_{2}$ for $k_{2}<k_{1}$. Thus, we get $\left|W\left(G_{1} \times G_{2}\right)\right|=\min \left\{k_{1}, k_{2}\right\}$. Such that, $\mu_{l}\left(G_{1} \times G_{2}\right) \geq \min \left\{\mu_{l}\left(G_{1}\right), \mu_{l}\left(G_{2}\right)\right\}$.

The cartesian product of graph $G$ and tree graph $T$ with characterization for $\mu_{l}(G)=1$ and we get the results as follows.

Theorem 3.1

Given that a connected graph $G$ and a path $P_{n}, \mu_{l}\left(G \times P_{n}\right)=\mu_{l}(G)$

Proof: The graph $G \times P_{n}$ has $n$ copies subgraph $G_{i}, 1 \leq i \leq n$. Let $W$ be a local resolving set of $G=G_{i}$ so that every vertices $u, v \in V(G)$ for $u$ adjacent to $v$ has different representation. If we assume that $W$ is a set of $G \times P_{n}$, then we prove that $W$ is local resolving set of $G \times P_{n}$,

i. We know that for every vertices $u \in W$ belong to in subgraph $G_{1}$ or first copy (first layer).

ii. Every two adjacent vertices $u, v \in V\left(G_{1}\right)-W$, has different representation. Since, a set $W$ is the local resolving set of $G=G_{1}$.

iii. For every two adjacent vertices $u \in V\left(G_{1}\right)-W$ and $v \in V\left(G_{j}\right)$ such that for $w \in W, d(u, w)=d^{\prime}$ and $d(v, w)=d(v, u)+d(u, w)=d(u, v)+d^{\prime}>d^{\prime}=d(u, w)$ which $d(u, w) \neq d(v, w)$. Thus, $r(u \mid W) \neq$ $r(v \mid W)$.

iv. For $G_{i}$ and $G_{j}, 1 \leq i<j \leq n$. Choose two adjacent vertices $u \in V\left(G_{i}\right)$ and $v \in V\left(G_{j}\right)$ such that for $w \in$ $W, d(u, w)=d(u, x)+d(x, w), \forall x \in V\left(G_{1}\right)$. We know that $i<j$ such that $d(v, w)=d(v, u)+d(u, x)+$ $d(x, w)>d(u, x)+d(x, w)=d(u, w)$ so $d(u, w) \neq d(v, w)$. Thus, $r(u \mid W) \neq r(v \mid W)$.

v. For every two adjacent vertices $u, v \in V\left(G_{i}\right)$ which have $d(u, w)=d(u, x)+d(x, w)$ and $d(v, w)=$ $d(v, y)+d(y, w)$ where $x, y, w \in V\left(G_{1}\right)$ such that $d(x, w) \neq d(y, w)$ and $d(u, x)=d(v, y)$. We have $d(u, w) \neq d(v, w)$. Thus, $r(u \mid W) \neq r(v \mid W)$.

Based on five cases $(i)-(v), W$ is a local resolving set of $G \times P_{n}$. Thus, we have upper bound of local multiset dimension of $G \times P_{n}$ is $\mu_{l}\left(G \times P_{n}\right) \leq \mu_{l}(G)$.

Furthermore, we show that the lower bound of local multiset dimension of $G \times P_{n}$ is $\mu_{l}\left(G \times P_{n}\right) \geq \mu_{l}(G)$. Assume that $\left|W_{G \times P_{n}}\right|<\left|W_{G}\right|$, by taking $\left|W_{G \times P_{n}}\right|=\left|W_{G}\right|-1$.

i. For every vertices $v \in W_{G \times P_{n}}$ belong to in subgraph $G_{1}$ such that there exists at least two adjacent vertices has same representation.

ii. Let $u, v \in V\left(G_{1}\right)$ where $u$ adjacent to $v, d(u, w)=d(v, w)$. Thus, $r(u \mid W)=r(v \mid W)$. It is a contradiction.

iii. If some vertices of resolving set not all in subgraph $G_{1}$, then there is at least one vertex of $W$ in $G_{i}, 1 \leq i \leq n$. 


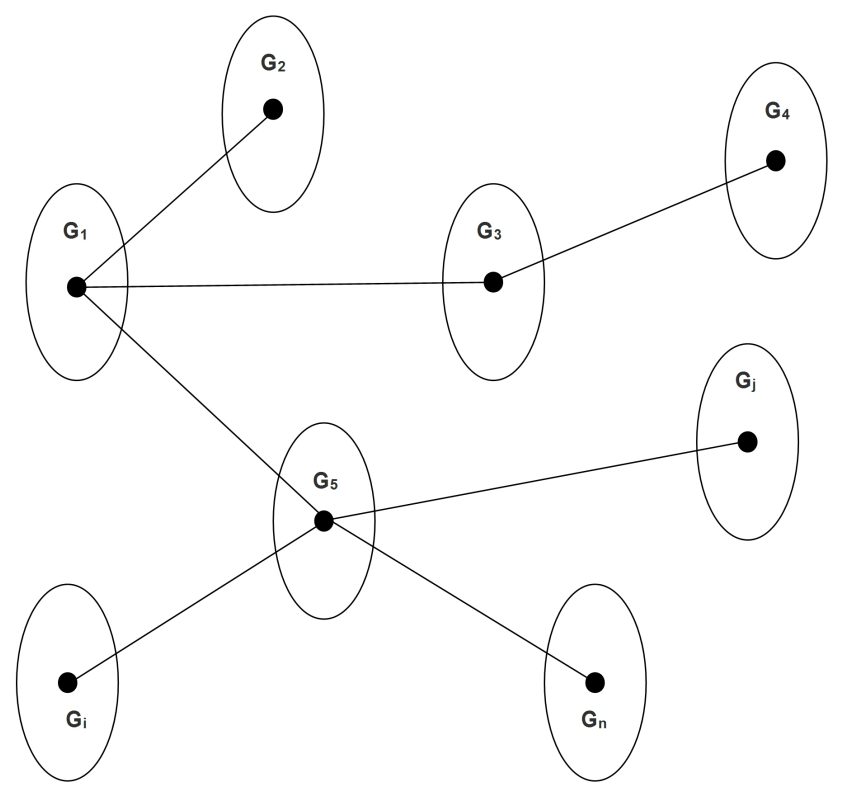

Figure 5. Illustration of $G \times T$

iv. For any two adjacent vertices $u, v \in G_{i}$, we have $d\left(u, w_{i}\right)=d\left(v, w_{i}\right)$ such that $d(u, w)=d(u, x)+d(x, w)$ and $d(v, w)=d(v, y)+d(y, w)$, we have $d(u, w)=d(v, w)$. Thus, $r(u \mid W)=r(v \mid W)$. It is a contradiction.

Based cases above, we have the local resolving set of $G \times P_{n}$ at least $\left|W_{G}\right|$ or $\left|W_{G \times P_{n}}\right| \geq\left|W_{G}\right|$. Hence, we have lower bound of local multiset dimension of $G \times P_{n}$ is $\mu_{l}\left(G \times P_{n}\right) \geq \mu_{l}(G)$. Thus, the local multiset dimension of $G \times P_{n}$ is $\mu_{l}\left(G \times P_{n}\right)=\mu_{l}(G)$.

\section{Theorem 3.2}

Given that a connected graph $G$ and a tree $T, \mu_{l}(G \times T) \leq \mu_{l}(G)$

Proof: The graph $G \times T$ has $n$ copies subgraph $G_{i}, 1 \leq i \leq n$. Let $W$ be a local resolving set of $G=G_{i}$ so that every vertices $u, v \in V(G)$ for $u$ adjacent to $v$ has different representation. If we assume that $W$ is a set of $G \times T$, then we prove that $W$ is local resolving set of $G \times T$,

i. We know that for every vertices $u \in W$ belong to in subgraph $G_{1}$ or first copy (first layer).

ii. Every two adjacent vertices $u, v \in V\left(G_{1}\right)-W$, has different representation. Since, a set $W$ is the local resolving set of $G=G_{1}$.

iii. For $G_{i}$ and $G_{j}, 1 \leq i<j \leq n$. Choose two adjacent vertices $u \in V\left(G_{i}\right)$ and $v \in V\left(G_{j}\right)$ such that for $w \in$ $W, d(u, w)=d(u, x)+d(x, w), \forall x \in V\left(G_{1}\right)$. We know that $i<j$ such that $d(v, w)=d(v, u)+d(u, x)+$ $d(x, w)>d(u, x)+d(x, w)=d(u, w)$ so $d(u, w) \neq d(v, w)$. Thus, $r(u \mid W) \neq r(v \mid W)$.

iv. For every two adjacent vertices $u, v \in V\left(G_{i}\right)$ which have $d(u, w)=d(u, x)+d(x, w)$ and $d(v, w)=$ $d(v, y)+d(y, w)$ where $x, y, w \in V\left(G_{1}\right)$ such that $d(x, w) \neq d(y, w)$ and $d(u, x)=d(v, y)$. We have $d(u, w) \neq d(v, w)$. Thus, $r(u \mid W) \neq r(v \mid W)$.

Based on four cases $(i)-(i v), W$ is a local resolving set of $G \times T$. Thus, we have upper bound of local multiset dimension of $G \times T$ is $\mu_{l}(G \times T) \leq \mu_{l}(G)$.

\section{Corollary 3.2}

Given that a connected graph $G$ and a tree $T$. For $\mu_{l}(G)=1, \mu_{l}(G \times T)=1$

Proof: Alfarisi, et. al. [8] determined the local multiset dimension of $T$ is 1 . If the local multiset dimension of $G$ is 1 , then every two adjacent vertices have distinct representation. 


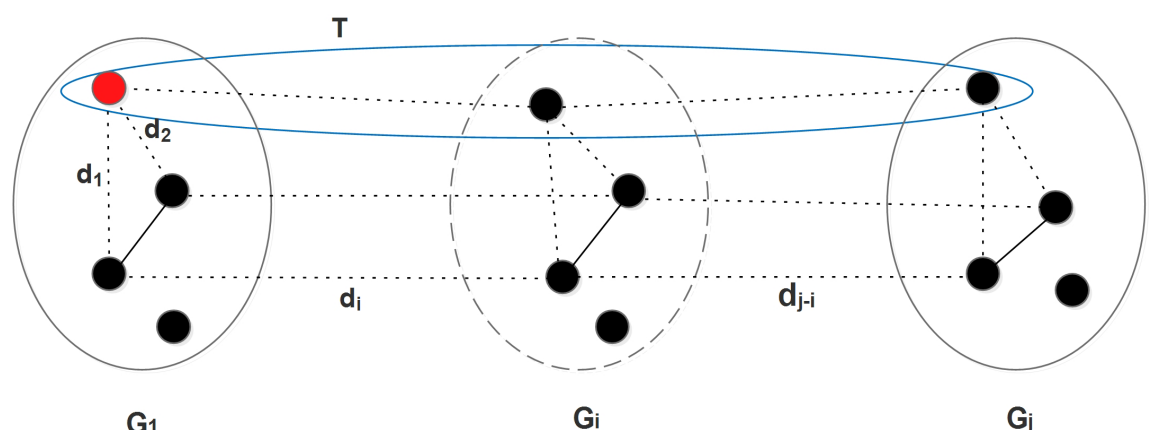

Figure 6. Illustration of $G \times T$ for $d_{1} \neq d_{2}$

From Lemma 3.2 that $\mu_{l}(G \times T) \geq \min \left\{\mu_{l}(G), \mu_{l}(1)\right\} \geq \min \{1,1\} \geq 1$. Furthermore, we can show $\mu_{l}(G \times$ $T) \leq 1$ as follows.

i. the graph $G \times T$ has $|V(T)|$-copies of subgraph $G$, namely $G_{j}, 1 \leq j \leq|V(T)|$

ii. we choose the local resolving set in first copy $G_{1}$, namely $W=\left\{\left(x_{1}, u^{*}\right)\right\}$

iii. we know that $\mu_{l}(G)=1$, the distance of two adjacent vertices in $\left(x_{1}, u_{k}\right),\left(x_{1}, u_{l}\right) \in V\left(G_{1}\right)-W$ is $d\left(\left(x_{1}, u_{k}\right),\left(x_{1}, u^{*}\right)\right) \neq d\left(\left(x_{1}, u_{l}\right),\left(x_{1}, u^{*}\right)\right)$ for $1 \leq k, l \leq V(G)$.

iv. every two adjacent vertices $\left(x_{j}, u_{k}\right),\left(x_{j}, u_{l}\right) \in V\left(G_{j}\right)$ has distinct representation, such that $d\left(\left(x_{j}, u_{k}\right),\left(x_{1}, u^{*}\right)\right) \neq d\left(\left(x_{j}, u_{l}\right),\left(x_{1}, u^{*}\right)\right)$ where $d\left(\left(x_{j}, u_{k}\right),\left(x_{1}, u^{*}\right)\right)=d\left(\left(x_{j}, u_{k}\right)\right.$,

$\left.\left(x_{1}, u_{k}\right)\right)+d\left(\left(x_{1}, u_{k}\right),\left(x_{1}, u^{*}\right)\right)$ and $d\left(\left(x_{j}, u_{l}\right),\left(x_{1}, u^{*}\right)\right)=d\left(\left(x_{j}, u_{l}\right),\left(x_{1}, u_{l}\right)\right)+d\left(\left(x_{1}, u_{l}\right)\right.$, $\left.\left(x_{1}, u^{*}\right)\right)$.

v. every two adjacent vertices $\left(x_{i}, u_{l}\right) \in V\left(G_{i}\right), \quad\left(x_{j}, u_{l}\right) \in V\left(G_{j}\right)$ has distinct representation, such that $\quad d\left(\left(x_{i}, u_{l}\right),\left(x_{1}, u^{*}\right)\right) \neq d\left(\left(x_{j}, u_{l}\right),\left(x_{1}, u^{*}\right)\right) \quad$ where $\quad d\left(\left(x_{i}, u_{l}\right),\left(x_{1}, u^{*}\right)\right)=d\left(\left(x_{i}, u_{l}\right),\left(x_{1}, u_{l}\right)\right)+$ $d\left(\left(x_{1}, u_{l}\right),\left(x_{1}, u^{*}\right)\right)$ and $d\left(\left(x_{j}, u_{l}\right),\left(x_{1}, u^{*}\right)\right)=d\left(\left(x_{j}, u_{l}\right),\left(x_{1}, u_{l}\right)\right)+d\left(\left(x_{1}, u_{l}\right),\left(x_{1}, u^{*}\right)\right)$.

Thus, we obtain $\mu_{l}(G \times T) \leq 1$. Thus, $\mu_{l}(G \times T)=1$, for $\mu_{l}(G)=1$ and any tree $T$.

\section{Lemma 3.3}

For $\mu_{l}(G) \neq 1$ and any tree $T, \mu_{l}(G \times T) \geq 2$.

Proof: If local multiset dimension $\mu_{l}(G) \neq 1$, then we have local resolving set $|W| \geq 2$. From Lemma 3.2, it states that $\mu_{l}(G \times T) \geq \min \left\{\mu_{l}(G), 1\right\} \geq 1$. Assume that $|W|=1$. There is at least two adjacent vertices which have same representation. Choose the local resolving set in $G_{1}$. Every adjacent vertices in $\left(x_{1}, u_{k}\right),\left(x_{1}, u_{l}\right) \in$ $V\left(G_{1}\right)-W$ has some distances, namely $d\left(\left(x_{1}, u_{k}\right),\left(x_{1}, u^{*}\right)=d\left(\left(x_{1}, u_{l}\right),\left(x_{1}, u^{*}\right)\right.\right.$. Thus, the cardinality of the local resolving set of $G \times T$ is $|W| \neq 1$, and the local multiset dimension of $\mu_{l}(G \times T) \geq 2$.

Theorem 3.3

For $\mu_{l}(G)=1$ and $m$ is even, $\mu_{l}\left(G \times C_{m}\right)=1$.

Proof: Alfarisi, et. al. [8] determined the local multiset dimension of $C_{m}$ with $m$ is odd is 1 . If the local multiset dimension of $G$ is 1 , then every two adjacent vertices has distinct representation.

From Lemma 3.2 that $\mu_{l}\left(G \times C_{m}\right) \geq \min \left\{\mu_{l}(G), \mu_{l}\left(C_{m}\right)\right\} \geq \min \{1,1\} \geq 1$. Furthermore, we can show $\mu_{l}\left(G \times C_{m}\right) \leq 1$ as follows.

i. the graph $G \times C_{m}$ has $\left|V\left(C_{m}\right)\right|$-copies of subgraph $G$, namely $G_{j}, 1 \leq j \leq\left|V\left(C_{m}\right)\right|$

ii. we choose the local resolving set in first copy $G_{1}$, namely $W=\left\{\left(x_{1}, u^{*}\right)\right\}$

iii. we know that $\mu_{l}(G)=1$, the distance of two adjacent vertices in $\left(x_{1}, u_{k}\right),\left(x_{1}, u_{l}\right) \in V\left(G_{1}\right)-W$ is $d\left(\left(x_{1}, u_{k}\right),\left(x_{1}, u^{*}\right)\right) \neq d\left(\left(x_{1}, u_{l}\right),\left(x_{1}, u^{*}\right)\right)$ for $1 \leq k, l \leq V(G)$. 


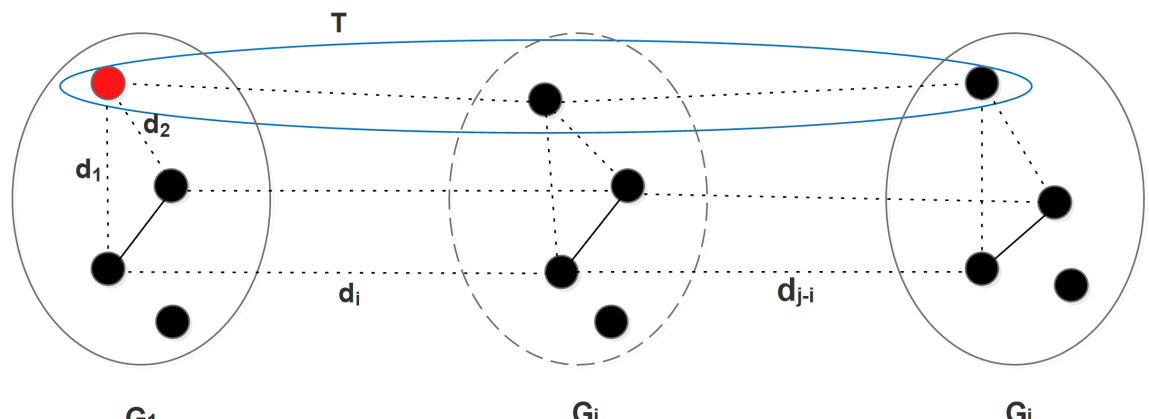

$\mathbf{G}_{1}$

$\mathbf{G}_{\mathrm{i}}$

$\mathbf{G}_{\mathrm{j}}$

Figure 7. Illustration of $G \times T$ for $d_{1} \neq d_{2}$

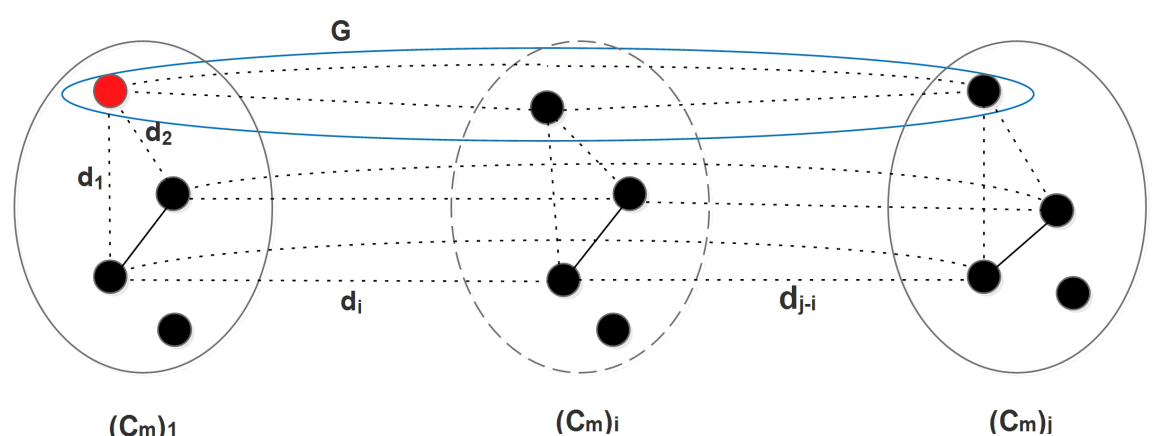

Figure 8. Illustration of $G \times C_{m}$ for $d_{1}=d_{2}$

iv. every two adjacent vertices $\left(x_{j}, u_{k}\right),\left(x_{j}, u_{l}\right) \in V\left(G_{j}\right)$ has distinct representation such that $d\left(\left(x_{j}, u_{k}\right),\left(x_{1}, u^{*}\right)\right) \neq d\left(\left(x_{j}, u_{l}\right),\left(x_{1}, u^{*}\right)\right)$ where $d\left(\left(x_{j}, u_{k}\right),\left(x_{1}, u^{*}\right)\right)=d\left(\left(x_{j}, u_{k}\right)\right.$, $\left.\left(x_{1}, u_{k}\right)\right)+d\left(\left(x_{1}, u_{k}\right),\left(x_{1}, u^{*}\right)\right)$ and $d\left(\left(x_{j}, u_{l}\right),\left(x_{1}, u^{*}\right)\right)=d\left(\left(x_{j}, u_{l}\right),\left(x_{1}, u_{l}\right)\right)+d\left(\left(x_{1}, u_{l}\right)\right.$, $\left.\left(x_{1}, u^{*}\right)\right)$.

v. every two adjacent vertices $\left(x_{i}, u_{l}\right) \in V\left(G_{i}\right), \quad\left(x_{j}, u_{l}\right) \in V\left(G_{j}\right)$ has distinct representation such that $\quad d\left(\left(x_{i}, u_{l}\right),\left(x_{1}, u^{*}\right)\right) \neq d\left(\left(x_{j}, u_{l}\right),\left(x_{1}, u^{*}\right)\right) \quad$ where $\quad d\left(\left(x_{i}, u_{l}\right),\left(x_{1}, u^{*}\right)\right)=d\left(\left(x_{i}, u_{l}\right),\left(x_{1}, u_{l}\right)\right)+$ $d\left(\left(x_{1}, u_{l}\right),\left(x_{1}, u^{*}\right)\right)$ and $d\left(\left(x_{j}, u_{l}\right),\left(x_{1}, u^{*}\right)\right)=d\left(\left(x_{j}, u_{l}\right),\left(x_{1}, u_{l}\right)\right)+d\left(\left(x_{1}, u_{l}\right),\left(x_{1}, u^{*}\right)\right)$.

Thus, we obtain that $\mu_{l}\left(G \times C_{m}\right) \leq 1$. It concludes that $\mu_{l}\left(G \times C_{m}\right)=1$, for $\mu_{l}(G)=1$ and $m$ is even.

\section{Lemma 3.4}

For $\left(\mu_{l}(G)=1\right.$ and $m$ is odd $)$ or $\left(\mu_{l}(G) \neq 1\right.$ and $\left.m \geq 3\right), \mu_{l}\left(G \times C_{m}\right) \geq 2$.

Proof: Based on Lemma 3.1 that $\mu_{l}\left(G_{1} \times G_{2}\right)=\min \left\{\mu_{l}\left(G_{1}\right), \mu_{l}\left(G_{2}\right)\right\}$. If one of both graph has local multiset dimension at least one, then $\mu_{l}\left(G \times C_{m}\right) \geq 1$. We try construct of the sharpest lower bound of $G \times C_{m}$ for $m$ is odd or $\left(\mu_{l}(G) \neq 1\right.$ and $m$ is even) as follows.

Case 1: For $\mu_{l}(G)=1$ and $m$ is odd

We know that $\mu_{l}\left(C_{m}\right)=3$ for $n$ is odd and $\mu_{l}(G)=1$, based Lemma 3.2 that $\mu_{l}\left(G \times C_{m}\right) \geq \min \{1,3\} \geq 1$. Assume that $|W|=1$, there is at least two adjacent vertices which have same representation. Choose the local resolving set in $\left(C_{m}\right)_{1}$, then every adjacent vertices in $\left(x_{1}, u_{k}\right),\left(x_{1}, u_{l}\right) \in V\left(\left(C_{m}\right)_{1}\right)-W$ has some distance namely $d\left(\left(x_{1}, u_{k}\right),\left(x_{1}, u^{*}\right)=d\left(\left(x_{1}, u_{l}\right),\left(x_{1}, u^{*}\right)\right.\right.$. Thus, the cardinality of the local resolving set of $G \times C_{m}$ is $|W| \neq 1$, such that the local multiset dimension of $\mu_{l}\left(G \times C_{m}\right) \geq 2$. This illustration can be seen in Figure 8

Case 2: For $\mu_{l}(G) \neq 1$ and $m$ is odd

If local multiset dimension $\mu_{l}(G) \neq 1$, then we have local resolving set $|W| \geq 2$. From Lemma 3.2 that $\mu_{l}(G \times$ 


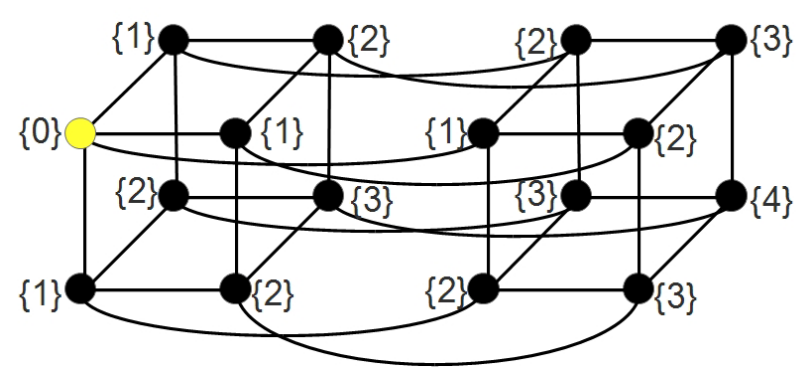

Figure 9. Illustration of $\mathrm{HQ}_{4}$

$\left.C_{m}\right) \geq \min \left\{\mu_{l}(G), 3\right\} \geq 2$. Assume that $|W|=1$ such that there is at least two adjacent vertices which have same representation. Choose the local resolving set in $G_{1}$, then every adjacent vertices in $\left(x_{1}, u_{k}\right),\left(x_{1}, u_{l}\right) \in V\left(G_{1}\right)-W$ have some distance namely $d\left(\left(x_{1}, u_{k}\right),\left(x_{1}, u^{*}\right)=d\left(\left(x_{1}, u_{l}\right),\left(x_{1}, u^{*}\right)\right.\right.$. Thus, the cardinality of the local resolving set of $G \times C_{m}$ is $|W| \neq 1$, such that the local multiset dimension of $\mu_{l}\left(G \times C_{m}\right) \geq 2$.

Case 3: For $\mu_{l}(G) \neq 1$ and $m$ is even

If local multiset dimension $\mu_{l}(G) \neq 1$, then we have local resolving set $|W| \geq 2$. From Lemma 3.2 that $\mu_{l}(G \times$ $\left.C_{m}\right) \geq \min \left\{\mu_{l}(G), 1\right\} \geq 1$. Assume that $|W|=1$ such that there is at least two adjacent vertices which have same representation. Choose the local resolving set in $G_{1}$, then every adjacent vertices in $\left(x_{1}, u_{k}\right),\left(x_{1}, u_{l}\right) \in V\left(G_{1}\right)-W$ have some distance namely $d\left(\left(x_{1}, u_{k}\right),\left(x_{1}, u^{*}\right)=d\left(\left(x_{1}, u_{l}\right),\left(x_{1}, u^{*}\right)\right.\right.$. Thus, the cardinality of the local resolving set of $G \times C_{m}$ is $|W| \neq 1$, such that the local multiset dimension of $\mu_{l}\left(G \times C_{m}\right) \geq 2$. The local multiset dimension of $G \times C_{m}$ is $\mu_{l}\left(G \times C_{m}\right) \geq 2$.

Next, we study a Hypercube graph, denoted by $H Q_{n}$. Hypercube graph is the graph formed from the vertices and edges of an $n$-dimensional hypercube. It is the $n$-fold Cartesian product of the two-vertex complete graph, and decomposed into two copies of $H Q_{n-1}$ connected to each other by a perfect matching.

Theorem 3.4

For $n \in N \cup\{0\}, \mu_{l}\left(H Q_{n}\right)=1$.

Proof: Hypercube graph satisfies $H Q_{n}=H Q_{n-1} \times P_{2}$ for $n \geq 0$. For $n=0$, we have $H Q_{0}$ isomorphic to $K_{1}$ or trivial graphs. The local multiset dimension of $K_{1}$ is $\mu_{l}\left(H Q_{0}\right)=1$. To prove this theorem, we can use a mathematical induction or recursive technique below.

i. For $n=1$, we have $H Q_{1}=H Q_{0} \times P_{2}$. Based on Lemma 3.2, it holds $\mu_{l}\left(H Q_{1}\right)=\mu_{l}\left(H Q_{0} \times P_{2}\right)=1$ since $\mu_{l}\left(H Q_{0}\right)=1$.

ii. For $n=2$, we have $H Q_{2}=H Q_{1} \times P_{2}$. Based on Lemma 3.2, it holds $\mu_{l}\left(H Q_{2}\right)=\mu_{l}\left(H Q_{1} \times P_{2}\right)=1$ since $\mu_{l}\left(H Q_{1}\right)=1$.

iii. Assume that for $n=k$, we have $H Q_{k}=H Q_{k-1} \times P_{2}$. Based on Lemma 3.2, it holds $\mu_{l}\left(H Q_{k}\right)=$ $\mu_{l}\left(H Q_{k-1} \times P_{2}\right)=1$ since $\mu_{l}\left(H Q_{k-1}\right)=1$.

iv. For $n=k+1$, we prove that $\mu_{l}\left(H Q_{k} \times P_{2}\right)=1$ ?. From point iii, we have $\mu_{l}\left(H Q_{k}\right)=1$. Recalling Lemma 3.2, It implies that $\mu_{l}\left(H Q_{k+1}\right)=\mu_{l}\left(H Q_{k} \times P_{2}\right)=1$ since $\mu_{l}\left(H Q_{k}\right)=1$.

Thus, the local multiset dimension of hypercube $H Q_{n}$ is $\mu_{l}\left(H Q_{n}\right)=1$, for $n \in N \cup\{0\}$. Figure 9 is an illustration of local multiset dimension of hypercube graphs for $H Q_{4}$.

Theorem 3.5

$\mu_{l}\left(A H Q_{n}\right)=1$, for $n \geq 2$ 


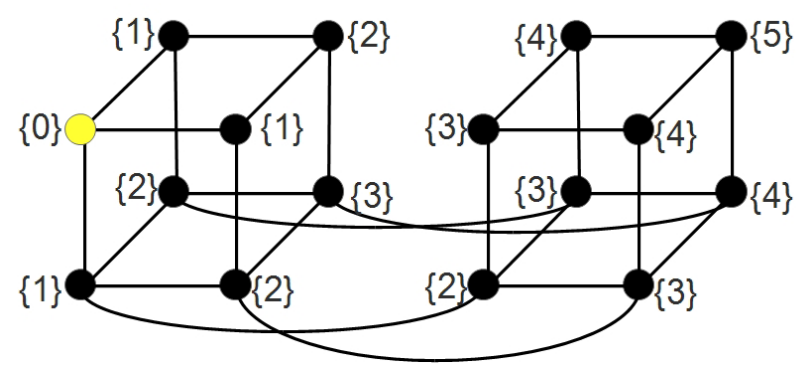

Figure 10. Illustration of $A H Q_{4}$

Proof: Almost hypercube graph satisfies $A H Q_{n}=\left(H Q_{n-1} \times P_{2}\right)-\{e\}$ for $n \geq 3$, where $e$ is correspondence edge of subgraph $\left(H Q_{n-1}\right)_{1}$ and $\left(H Q_{n-1}\right)_{2}$. We know that $H Q_{n-1} \times P_{2}$ has two isomorphic graphs $\left(H Q_{n-1}\right)_{1}$ and $\left(H Q_{n-1}\right)_{2}$ with $\{e\}$ is the correspondence edge set. Based on Theorem 3.4 that $\mu_{l}\left(H Q_{n-1}\right)=1$ such that for two adjacent vertices $u, v \in V\left(\left(H Q_{n-1}\right)_{1}\right)$ has the distinct representation namely $r(u \mid W) \neq r(v \mid W)$ for $W$ in the first copy of $H Q_{n-1}$.

i. If we choose two adjacent vertices in $u, v \in V\left(\left(H Q_{n-1}\right)_{2}\right)$ with their vertices are correspondence by edge to two adjacent vertices in $x, y \in V\left(\left(H Q_{n-1}\right)_{1}\right)$ where $d(x, w) \neq d(y, w)$ for $w \in W$. We have the distance $d(u, x)=d(v, y)=1$ and $d(u, w)=d(u, x)+d(x, w)=1+d(x, w), d(v, w)=d(v, y)+$ $d(y, w)=1+d(y, w)$ then $d(u, w) \neq d(v, w)$. Thus, we have $r(u \mid W) \neq r(v \mid W)$.

ii. If we choose two adjacent vertices in $u, v \in V\left(\left(H Q_{n-1}\right)_{2}\right)$ with their vertices are not correspondence by edge to two adjacent vertices in $x, y \in V\left(\left(H Q_{n-1}\right)_{1}\right)$ where $d(x, w) \neq d(y, w)$ for $w \in W$. But We have the distance $d(u, x)>1, d(v, y)>1$ and $d(u, w)=d(u, x)+d(x, w)=d+d(x, w), d(v, w)=d(v, y)+$ $d(y, w)=d^{*}+d(y, w)$ then $d(u, w) \neq d(v, w)$. Thus, we have $r(u \mid W) \neq r(v \mid W)$.

iii. If we choose two adjacent vertices in $u, v \in V\left(\left(H Q_{n-1}\right)_{2}\right)$ with one of them are correspondence by edge to adjacent vertices in $x \in V\left(\left(H Q_{n-1}\right)_{1}\right)$ for $w \in W$. But we have the distance $d(u, x)=1, d(v, x)=d(u, v)+$ $d(v, x)=1+1=2 \quad$ and $d(u, w)=d(u, x)+d(x, w)=1+d(x, w), \quad d(v, w)=d(v, y)+d(y, w)=2+$ $d(y, w)$ then $d(u, w) \neq d(v, w)$. Thus, we have $r(u \mid W) \neq r(v \mid W)$.

Based on three cases above, the local resolving set of almost hypercube graphs $|W|=1$. Thus, the local multiset dimension of almost hypercube graphs $A H Q_{n}$ is $\mu_{l}\left(A H Q_{n}\right)=1$, for $n \geq 3$. Figure 10 is an illustration of local multiset dimension of almost hypercube graphs for $A H Q_{4}$.

Kautz graphs $K(d, n)$ for $d \geq 2$ and $n \geq 2$, is defined as follows. The vertex set of $K(d, n)$ is $V(K(d, n))=$ $\left\{x_{1}, x_{2}, \ldots, x_{n} \mid x_{i} \in\{0,1,2, \ldots, d\}, x_{i} \neq x_{i+1}, i=1,2, \ldots, n-1\right\}$ and the edge set $E(K(d, n))$ consists of all edges from one vertex $x_{1}, x_{2}, \ldots, x_{n}$ to $d$ order vertices $x_{1}, x_{2}, \ldots, x_{n}, \alpha$, where $\alpha \in\{1,2, \ldots, d\}$ and $\alpha \neq x_{n}$. It is clear that $K(d n)$ is d-regular, $|V(d, n)|=d^{n}+d^{n 1}$ and $|E(d n)|=d^{n+1}+d^{n}$. Moreover, $K(d, n)$ has $\frac{d(d+1)}{2}$ pairs of symmetric edges. The Kautz undirected graph, denoted by $U K(d, n)$, is an undirected graph obtained from $K(d, n)$ by deleting the orientation of all edges and omitting multiple edges. It is clear that $U K(d n)$ has $d^{n+1}+d^{n}-\frac{d(d+1)}{2}$ edges, the maximum degree is $2 d$ for $n \geq 3$ and the minimum degree is $2 d-1$ for $n \geq 2$.

The Kautz undirected graph of cycle, denoted by $U K\left(C_{n}\right)$ is connected graphs with 3regular isomorphic to $C_{n} \times P_{2}$. The vertex set and edge set of $C_{n}$ respectively, are $V\left(C_{n}\right)=$ $\left\{x_{1}, x_{2}, \ldots, x_{n}\right\}$ and $E\left(C_{n}\right)=\left\{x_{1} x_{2}, x_{2} x_{3}, \ldots, x_{n-1} x_{n}, x_{n} x_{1}\right\}$. The vertex set of $U K\left(C_{n}\right)$ is $V\left(U K\left(C_{n}\right)\right)=\left\{x_{i,(i+1) \bmod n}, x_{(i+1) \bmod n, i}: 1 \leq i \leq n\right\} \quad$ and $\quad$ edge $\quad$ set $\quad$ of $\quad U K\left(C_{n}\right)$ is $E\left(U K\left(C_{n}\right)\right)=$ $\left\{x_{i,(i+1) \bmod n} x_{(i+1) \bmod n, i}, x_{i,(i+1) \bmod n} x_{(i+1) \bmod n,(i+2) \bmod n}, x_{(i+1) \bmod n, i} x_{(i+2) \bmod n,(i+1) \bmod n} ; 1 \leq i \leq n\right\}$.

\section{Corollary 3.3}

For $n \geq 4$,

$$
\mu_{l}\left(U K\left(C_{n}\right)\right)= \begin{cases}1, & \text { if } n \text { is even } \\ 3, & \text { if } n \text { is odd }\end{cases}
$$


Furthermore, we characterization of relationship between multiset dimension and local multiset dimension of graphs.

Remark 3.1

The relationship of multiset dimension and local multiset dimension of graphs $G, \operatorname{gap}\left(\operatorname{md}(G), \mu_{l}(G)=\infty\right.$.

\section{Conclusion}

In this paper we have given an result the lower bound of multiset dimension and local multiset dimension of graphs. Hence the following problem aries naturally.

Open Problem 4.1

Determine the local multiset dimension of family graph namely family tree, unicyclic, regular graphs, and others.

Open Problem 4.2

Determine the local multiset dimension of operation graph namely corona product, joint, comb product, and others.

\section{Acknowledgments}

We gratefully acknowledge the support from PIU-IsDB Project-University of Jember of year 2018.

\section{REFERENCES}

1. J. L. Gross, J. Yellen, and P. Zhang, Handbook of graph Theory Second Edition CRC Press Taylor and Francis Group, 2014.

2. G. Chartrand and L. Lesniak, Graphs and digraphs 3rd ed (London: Chapman and Hall), 2000.

3. N. Hartsfield and G. Ringel, Pearls in Graph Theory Academic Press. United Kingdom, 1994.

4. S. Khuller, B. Raghavachari and A. Rosenfeld, Localization in Graphs, Technical Report CS-Tr-3326, University of Maryland at College Park, 1994.

5. P. J. Slater, Leaves of trees, in:Proc. 6th Southeast Conf. Comb., Graph Theory, Comput. Boca Rotan, vol. 14, pp 549-559, 1975.

6. F. Harary and R. A. Melter, On The metric dimension of a graph, Ars Combin, vol. 2, pp 191-195, 1976.

7. R. Simanjuntak, T. Vetrik and P. B. Mulia, The multiset dimension of graphs, arXiv preprint arXiv:1711.00225, 2017.

8. R. Alfarisi, Dafik, A. I. Kristiana, I. H. Agustin, Local multiset dimension of graphs, Preprint, 2018.

9. S. W. Saputro, A. Semanicova-Fenovcikova, M. Baca, M. Lascsakova, On fractional metric dimension of comb product graphs, STATISTICS, OPTIMIZATION AND INFORMATION COMPUTING, vol.6, pp. 150-158, 2018.

10. R. Adawiyah, Dafik, I. H. Agustin, R. M. Prihandini, R. Alfarisi and E. R.Albirri, On the local multiset dimension of m-shadow graph, Journal of Physics: Conference Series, vol. 1211, p. 012006, 2019.

11. R. Adawiyah, Dafik, I. H. Agustin, R. M. Prihandini, R. Alfarisi and E. R.Albirri, The local multiset dimension of unicyclic graph, IOP Conference Series: Earth and Environmental Science, vol. 243, p. 012075, 2019. 